\title{
Electric vehicle charging station planning with dynamic prediction of elastic charging demand: a hybrid particle swarm optimization algorithm
}

\author{
Xingzhen Bai ${ }^{1} \cdot$ Zidong Wang $^{2} \cdot$ Lei Zou $^{2} \cdot$ Hongjian Liu $^{3,4}{ }^{1} \cdot$ Qiao Sun $^{1} \cdot$ Fuad E. Alsaadi $^{5}$
}

Received: 16 August 2021 / Accepted: 19 October 2021 / Published online: 10 November 2021

(c) The Author(s) 2021

\begin{abstract}
This paper is concerned with the electric vehicle (EV) charging station planning problem based on the dynamic charging demand. Considering the dynamic charging behavior of EV users, a dynamic prediction method of EV charging demand is proposed by analyzing EV users' travel law via the trip chain approach. In addition, a multi-objective charging station planing problem is formulated to achieve three objectives: (1) maximize the captured charging demands; (2) minimize the total cost of electricity and the time consumed for charging; and (3) minimize the load variance of the power grid. To solve such a problem, a novel method is proposed by combining the hybrid particle swarm optimization (HPSO) algorithm with the entropy-based technique for order preference by similarity to ideal solution (ETOPSIS) method. Specifically, the HPSO algorithm is used to obtain the Pareto solutions, and the ETOPSIS method is employed to determine the optimal scheme. Based on the proposed method, the siting and sizing of the EV charging station can be planned in an optimal way. Finally, the effectiveness of the proposed method is verified via the case study based on a test system composed of an IEEE 33-node distribution system and a 33-node traffic network system.
\end{abstract}

Keywords Electric vehicles (EVs) - Charging demand prediction · Trip chain · Charging station planning · Hybrid particle swarm optimization (HPSO)

Hongjian Liu

hjliu1980@gmail.com

Xingzhen Bai

xzbai@163.com

Zidong Wang

Zidong.Wang@brunel.ac.uk

1 College of Electrical Engineering and Automation, Shandong University of Science and Technology, Qingdao 266590, China

2 Department of Computer Science, Brunel University London, Uxbridge, Middlesex UB8 3PH, UK

3 Key Laboratory of Advanced Perception and Intelligent Control of High-end Equipment, Ministry of Education, Anhui Polytechnic University, Wuhu 241000, China

4 School of Mathematics and Physics, Anhui Polytechnic University, Wuhu 241000, China

5 Department of Electrical and Computer Engineering, Faculty of Engineering, King Abdulaziz University, Jeddah 21589, Saudi Arabia

\section{Introduction}

Due to the shortage of resources and increasing pressure on the environment protection, the past decades have witnessed a surge of research interest in energy-saving and emission reduction (such as event-triggered mechanism [10,11,22,24], transmission scheduling scheme [20,48] and energy-efficient sampling scheme [29]). With this background, electric vehicles (EVs) have become the future direction of the automotive industry development [40]. In addition, the continuous progress of battery technology and the incentive policies put forward by the governments have significantly facilitated the rapid development of EVs. It is well known that charging infrastructures (e.g. charging stations) are the essential connection between EVs and the power distribution grid [44]. As such, the reasonable planning of charging stations can not only provide the satisfactory charging service for EV users, but also adjust the load distribution efficiently to guarantee the stable and safe operation of power system [42].

The planning of EV charging stations has been capturing constant attention from both academy and industry, and 
a variety of planning schemes have been available in the existing literature. One of the main streams of research is to plan the layout of charging stations from the economic point of view $[5,14,30]$, and minimize the cost using certain optimization algorithms [26,27]. For instance, in [5], an optimal model has been proposed to minimize the investment and operation cost of the EV charging stations. A multi-objective optimization model for the charging station planning in residential areas has been established in [30] to minimize the total cost of line installation and power losses of the distribution system. In recent years, the road traffic information has also been incorporated in planning charging stations $[33,39,46]$. Specifically, a multi-objective decision-making model has been considered in [39] for the optimal planning of EV charging stations, aiming at maximizing the captured traffic network flow and minimizing the power network loss. In [33], a dynamic traffic network method has been used to minimize the capital cost and charging cost, thereby obtaining the optimal distribution and scale of EV charging station. It is worth mentioning that, so far, most reported results have been focused on the planning models that are formulated simply from the perspective of the economics and traffic flow information, and the EV charging demand has not been paid adequate research attention, which would seriously affect the rationality of the planned charging stations.

It is obvious that the charging demand is closely related to the travel characteristics of EV users. As such, the charging demand should be forecasted based on a comprehensive analysis of EV users' travel behaviours [3,39]. In [3], the planning method of EV charging station has taken into account the EV users' travel rules that are described by the trip chain. Moreover, by virtue of the Monte Carlo stochastic simulation of trip chain, a novel method has been proposed in [39] to analyze the dynamic charging demand for EVs. More specifically, the complete trip chains can be formed by extracting samples from the Monte Carlo simulation, and the probability distribution of characteristic variables in the trip chains can be analyzed and fitted. Then, it is possible to derive the charging demand distribution in the functional region with the full analysis of the EVs' travel characteristics.

In order to better integrate EVs into the power grid, it is quite necessary to consider the effect from the charging station layout on the operation of power grid $[16,17]$. Recently, the disordered charging has become an increasingly prominent problem as the rapid popularisation of EVs, which would lead to the load peak and do harm to the normal operation of power grid. To avoid such an issue, the load scheduling problem has been studied in [16] to minimize the serious peak-to-valley difference in the distribution grid and the financial loss. The real-time scheduling strategies have been proposed in some literature, see e.g. $[9,47]$, in which the timeof-use (TOU) electricity price has been used as an incentive measure to instruct users to charge during the off-peak peri- ods. Furthermore, based on the forecasted load information, an optimal EV charging scheduling model has been established in [35], where the relationships between feeder losses, load factor, and load variance have been revealed. In [13], an optimal charging time scheduling model has been developed to minimize the power cost of the power system. Obviously, the EV users' charging behavior would be affected by the scheduling policies, and the users are more willing to satisfy their charging demands with lower extra costs.

Motivated by the above discussions, in this paper, we aim to provide an optimal planning method of EV charging stations by taking into account the charging behavior of the EV users. To this end, a charging demand forecasting model is established and the charging station planning is formulated as a multi-objective optimization problem. In addition, the hybrid particle swarm optimization (HPSO) algorithm is utilized to solve the multi-objective optimization problem and obtain the Pareto solutions. Moreover, the entropy-based technique for order preference by similarity to ideal solution (ETOPSIS) method is used to determine the optimal locations of charging stations, and then the optimal sizes of the selected charging stations are derived. The main contributions of this paper are summarized as follows.

1. Based on the analysis of EV users' travel activities, a dynamic forecasting method of charging demand is presented with the consideration of the relationship between EV charging demands and individual driving habits.

2. A charging station planning model is proposed by taking the mutual coupling relationship between charging demand distribution and charging station layout into account, which aims to maximize the captured charging demands, minimize the total cost of electricity and the time consumed for charging, and minimize the load variance of the power grid.

3. A multi-objective optimization method is proposed to get the optimal planning scheme of EV charging stations in the designated functional region.

The rest of this paper is organized as follows. In the next section, the dynamic charging behavior of the EV users is analyzed, and the charging demand is forecasted. The multi-objective model for the optimal planning and the configuration model of EV charging station are formulated in the subsequent section followed by which the HPSO algorithm and the ETOPSIS method are presented to get the optimal siting of charging stations, and the optimal sizing of the corresponding stations are obtained. A case study is provided in the penultimate section based on a test system consisting of an IEEE 33-node distribution system and a 33-node traffic network system. Finally, the conclusions are drawn. 


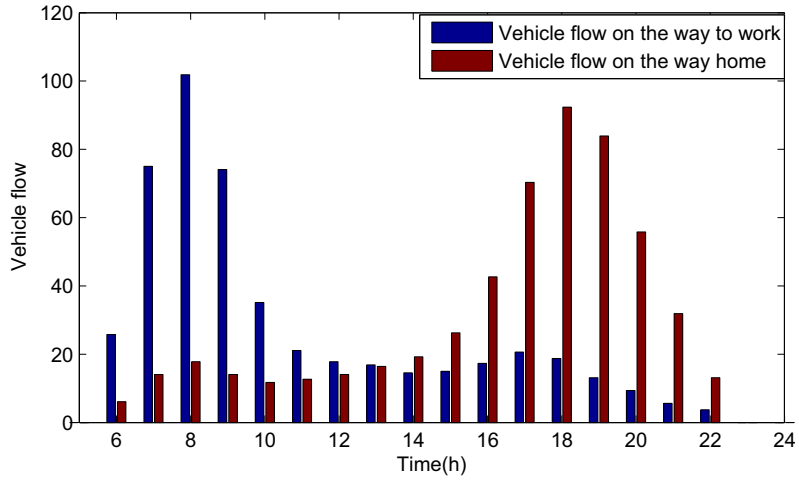

Fig. 1 Vehicle flow on the way to work and home

\section{Dynamic EV charging demand forecasting}

\section{Charging demand distribution and trip chain of EVs}

For the EV charging station planning, the first thing to focus on is the spatial and temporal distributions of charging demand. In practice, it is often the case that there is considerable distance between place of residence and employment location, which gives rise to the tide phenomenon at rush hours, and this could trigger off the large-scale, long-distance and tidal traffic flows [41], as shown in Fig. 1. During peak periods, there would be a surge of demand for fast charging on the way to and from work, while more conventional charging demands need to be fulfilled around the residential areas during off-peak hours [15]. ${ }^{1}$ In this case, the charging stations near the residential areas should be deployed with a higher proportion of conventional chargers, and the charging stations close to the commercial and industrial areas should be equipped with more fast chargers.

It is worth mentioning that the charging demand is closely related to EV users' travel law. The travel activities of EV can be described by the trip chain that is consistent with people's daily travel law. The trip chain is generally made by linking home and non-home sites, which includes the information of time, space and activity type during the trip [39]. Moreover, since the real-time state of charge (SOC) of EV is greatly significant for judging whether EV needs to be charged or not, the SOC of EV during travel process is added to the trip chain in this paper. As shown in Fig. 2, the circle represents the arriving/leaving time of each destination along the journey, the rectangle denotes the origin/destination position, and the hexagon represents the SOC of EV. Moreover, $T_{S_{-} 0}$ is the start time of the travel, $T_{a \_i}$ and $T_{S_{-} i}$ are, respectively, the arriving time and leaving time of destination $i . t_{p_{-} i}$ is the parking time at destination $i$, and $d_{i-1, i}$ is the distance from destination $i-1$ to destination $i$. $\mathrm{SOC}_{S_{-} 0}$ is the initial avail-

\footnotetext{
${ }^{1}$ Generally, the charging modes of EVs can be divided into two categories: the fast charging mode and conventional charging mode.
}

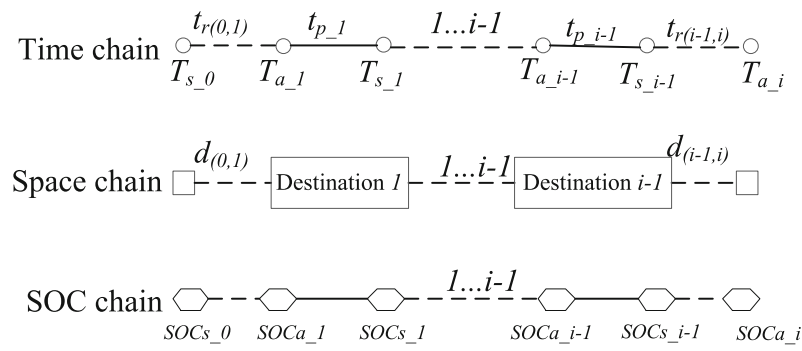

Fig. 2 Schematic diagram of the EV trip chain

able capacity, $\mathrm{SOC}_{a \_i}$ denotes the capacity when EV arrives at destination $i$, and $\mathrm{SOC}_{s_{-} i}$ denotes the capacity when $\mathrm{EV}$ leaves destination $i$.

\section{Dynamic forecasting of EV charging demand}

In general, the selection of charging station depends on a variety of factors which include, but are not limited to, the extra driving distance, road traffic conditions and the service capability of the alternative charging station. For the fast charging demand, the EV user usually focuses more on the extra driving distance and queuing time at the charging station. In contrast, the EV user with the conventional charging demand is more willing to choose the nearest charging station. As such, the satisfaction of EV user $k$ with the charging trip to station $j$ can be expressed by

$M_{k, j}(t)=$ $\left\{\begin{array}{l}\omega \frac{C_{\text {avg }}}{C_{k, j}^{e}(t)}+(1-\omega) \frac{F_{\max }}{F_{j}(t)}, \text { Selecting fast charging mode; } \\ \frac{L_{a v g}}{L_{O j}^{k}}, \quad \text { Selecting conventional charging mode. }\end{array}\right.$

where $C_{k, j}^{e}(t)$ denotes the extra charging cost of user $k$ at the station $j, C_{a v g}$ denotes the average charging cost, $F_{j}(t)$ denotes the time-varying vehicle flow at the charging station $j, F_{\max }$ denotes the maximum number of EVs waiting for charging at station $j$ [3], and $\omega$ is the compromise coefficient. In addition, $L_{O j}^{k}$ denotes the distance from the origin of user $k$ to the charging station $j$, and $L_{\text {avg }}$ denotes the average distance from the origins of $\mathrm{EV}$ users to the charging stations.

Moreover, the extra cost is defined as the sum of the extra electricity and time costs for charging, which can be mathematically formulated as follows:

$C_{k, j}^{e}(t)=q(t) E_{a} L_{k, j}^{e}+\alpha \beta(t) \frac{L_{k, j}^{e}}{v_{a}}$,

where $q(t)$ is the time-varying charging price, $E_{a}$ is the average capacity consumption per kilometer, $\alpha$ is the conversion coefficient of time cost, $\beta(t)$ is the traffic congestion coefficient, and $v_{a}$ is the average velocity of $\mathrm{EV}$. In addition, $L_{k, j}^{e}$ 
is the extra driving distance for EV user $k$ incurred by the charging trip to station $j$, which is given by

$L_{k, j}^{e}=L_{O j}^{k}+L_{j D}^{k}-L_{O D}^{k}$,

where $L_{O j}^{k}$ is the distance from origin $O$ to charging station $j$, $L_{j D}^{k}$ is the distance from charging station $j$ to destination $D$, and $L_{O D}^{k}$ is the direct distance from origin $O$ to destination $D$.

Now, we are in a position to present the dynamic forecasting method of EV charging demand, which is shown in Fig. 3. First, initialize the number of EVs and the characteristic variables (e.g. the trip purpose, starting time, origin, destination and initial SOC information). Second, the Monte Carlo sampling method is utilized to simulate the travel law of EV users to generate the trip chains. Thirdly, the trip chains are extracted according to the time sequence, and then the EV users' charging behaviors are analyzed one by one. With the initial travel information, such as the origin, destination and SOC of EVs, the maximum travel distance that each EV can run under the initial capacity is calculated, and the shortest distance between origin and destination is calculated by using the Dijkstra algorithm [45]. Comparing the two distances, we are able to make a judgment about whether or not the EV needs to be charged. If the EV needs to be charged, the EV user will select the charging station according to the satisfaction function defined in (1). If not, the user will drive directly to the destination. Finally, the number of EVs charging at the charging station in each time period and the charging loads would be counted to realize the dynamic forecasting of EV charging demand.

Remark 1 In this paper, in order to describe EV users' travel characteristics, the trip chain approach is adopted to analyze the spatial and temporal distributions of charging demands, which can accurately simulate the EV users' charging behavior during the travel process. In addition, the proposed satisfaction function is helpful to instruct EV users to choose charging station for the next charging cycle. It can be seen from (1) that the higher the satisfaction degree, the higher the possibility that the charging station can be selected. Hence, the proposed method is able to facilitate the efficient coordination of EV charging demand in the functional area, and make the charging station planning more reasonable.

\section{Charging station planning model formulation}

\section{Model for siting of EV charging station}

As a service provider, the charging station should satisfy EV users' charging requirements to the greatest extent. On

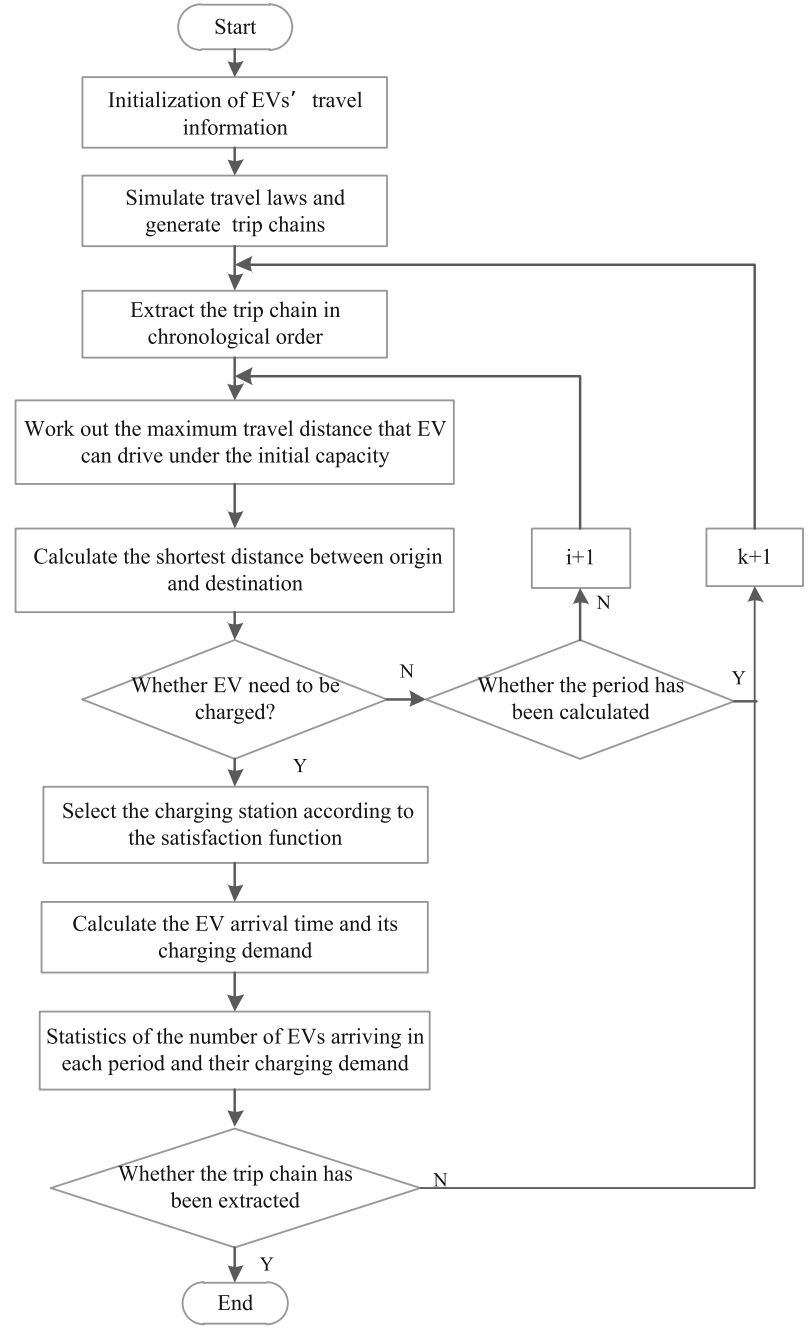

Fig. 3 Flow chart of EV charging demand forecasting

the other hand, as one of the important loads connected to the power distribution grid, the EV charging behavior may incur a variety of adverse effects on the power system, which include, but are not limited to, increased peak load, disrupted load balance, and local voltage deviation of the power grid [34]. Therefore, the planning of EV charging station is required to fully consider not only the charging demand, but also the impact of charging load on the power grid. To this end, in this subsection, a multi-objective charging station planning model is established to achieve the following three objectives: (1) maximize the captured charging demands; (2) minimize the extra electricity and time costs; and (3) minimize the load variance. The planning objectives and constraints are detailed as follows.

\section{(1) Maximizing the captured charging demands}

To meet the charging requirements of more EV users, the layout of charging stations should be able to cover the charging demand as much as possible. In addition, due to the 
mobility of EVs and the randomness of charging behaviors, the dynamicity of the EV charging demand should be incorporated in planning charging stations. Consequently, the first optimization objective is to maximize the sum of the charging demand divided by the extra driving distance. The specific mathematical expression is presented as follows:

Maximize : $Z_{1}=\sum_{j=1}^{\bar{n}} \sum_{k=1}^{m} Q_{k, j} R_{k}$

Subject to :

$R_{k} \leq c_{k j} x_{j}, j \in\{1,2, \ldots \bar{n}\}$

$\sum_{j} c_{k j} \leq 1, c_{k j} \in\{0,1\}, j \in\{1,2, \ldots \bar{n}\}$

$\sum_{j} x_{j}=n, x_{j} \in\{0,1\}, j \in\{1,2, \ldots \bar{n}\}$,

where $Q_{k, j}$ is the charging demand of EV user $k$ at charging station $j ; R_{k}$ is a binary variable: $R_{k}=1$ if the charging demand of EV user $k$ can be satisfied, and $R_{k}=0$ otherwise; $x_{j}$ is a binary variable: $x_{j}=1$ if an EV charging station is located at node $j$, and $x_{j}=0$ otherwise; $c_{k j}$ is a binary variable: $c_{k j}=1$ if EV $k$ can be charged at charging station $j$, and $c_{k j}=0$ otherwise; $n$ is the number of charging stations to be constructed; $\bar{n}$ is the total number of alternative location nodes for the charging station planning; and $m$ is the number of EVs in the functional region.

(2) Minimizing the extra cost of electricity and time consumed for charging

Generally speaking, EV users are eager to choose a charging station along their trip routes with lower charging cost. In order to minimize the extra cost of electricity and time consumed during the travel to the charging station, the second objective function is given as follows:

Minimize : $Z_{2}=\sum_{j=1}^{n} \sum_{k=1}^{m} C_{k, j}^{e}(t) R_{k}$,

where $C_{k, j}^{e}(t)$ denotes the extra cost of EV user $k$ consumed for charging at station $j$, which is defined in (2).

\section{(3) Minimizing the load variance of power grid}

It should be noted that the soaring charging demand may give rise to a new peak form during the normal off-peak period, thus the scheduling policies are usually utilized to guide the EV users' charging behaviors [36]. To minimize the load variance and smooth the load fluctuation of power grid, the third optimization objective is proposed as follows:

Minimize $: Z_{3}=\frac{1}{T} \sum_{t=1}^{T}\left[\left(P_{\text {grid }, t}+\sum_{j=1}^{n} P_{j, t}\right)-\right.$

$$
\left.\frac{1}{T} \sum_{s=1}^{T}\left(P_{g r i d, s}+\sum_{j=1}^{n} P_{j, s}\right)\right]^{2}
$$

Subject to : $0 \leq P_{j, t} \leq \zeta P_{j}^{\max }$,

where $T$ denotes the number of calculation periods, $P_{\text {grid, } t}$ denotes the predicted value of the power grid load, $P_{j, t}$ is the predicted value of the charging load at the station $j, P_{j}^{\max }$ is the maximum charging power that can be provided by charging station $j$, and $\zeta$ is the charging efficiency.

\section{Model for sizing of EV charging station}

As mentioned in section "Dynamic EV charging demand forecasting", there are usually two service modes available for different charging requirements, namely, fast charging mode and conventional charging mode, and the corresponding principles of charging station capacity allocation are different. As for the fast charging, the more chargers are equipped in the charging station, the less time EV users would consume for waiting. Nevertheless, to save the construction cost of the charging station, the operators are generally less willing to deploy more chargers, which will inevitably affect the charging efficiency. Therefore, aiming to minimize both the charging station construction cost and EV users' queuing time, the objective function is defined as follows:

$$
\begin{aligned}
\text { Minimize }: C_{f}= & C_{c}+C_{q} \\
= & \left(a_{f}+b_{f}\right) c h_{f, j} \frac{r(1+r)^{\tau}}{(1+r)^{\tau}-1} \\
& +365 \sum_{k}^{m} \alpha W_{k, j},
\end{aligned}
$$

where

$W_{k, j}=\frac{\left(c h_{f, j} \cdot \rho\right)^{c h_{f, j}} \cdot \rho}{c h_{f, j} !(1-\rho)^{2} \lambda} P_{0}$

$P_{0}=\left[\sum_{h=0}^{c h_{f, j}-1} \frac{1}{h !}\left(\frac{\lambda}{\mu}\right)^{h}+\frac{1}{c h_{f, j} !} \cdot \frac{1}{1-\rho} \cdot\left(\frac{\lambda}{\mu}\right)^{c h_{f, j}}\right]^{-1}$,

$C_{f}$ denotes the sum of charging station construction cost and EV users' queuing time cost, $C_{c}$ is the charging station construction cost, and $C_{q}$ is $\mathrm{EV}$ user's queuing time cost; $a_{f}$ is the unit price of the charger, $b_{f}$ is the operation and maintenance cost of a single charger, $c h_{f, j}$ is the number of fast chargers equipped in the charging station $j, r$ is the 
discount rate, and $\tau$ is the operating years; $W_{k, j}$ is the waiting time of $\mathrm{EV}$ user $k$ at charging station $j, P_{0}$ is the probability that all chargers are idle, and $\alpha$ is the conversion coefficient; and $\rho=\lambda / \mu$ is the service strength of chargers, where $\lambda$ is the number of EVs reaching the charging station and $\mu$ is the average service rate of chargers.

It is clear that the $\operatorname{cost} C_{f}$ is a function related to the number of chargers. As the number of chargers increases, the waiting time of EV users gradually decreases, while the construction cost of charging stations gradually increases. The optimal number of chargers equipped in the charging station can be determined by solving the optimization problem described by (9).

On the other hand, the EV users, who choose the conventional charging mode, prefer shorter waiting time. The conventional charging mode usually adopts the constant charging power to charge the EVs, and the optimal number of chargers can be calculated based on the largest charging demand in one day as follows:

$c h_{c, j}=\frac{Q_{c, j}^{\max }}{P_{a} T_{j} \tilde{\mu} \delta_{j}}$,

where $c h_{c, j}$ denotes the number of conventional chargers equipped in charging station $j, Q_{c, j}^{\max }$ represents the largest conventional charging demand in one day, $P_{a}$ is the charging power of a single charger, $\tilde{\mu}$ stands for the charging rate, and $\delta_{j}$ denotes the coincidence factor of charging station $j$.

\section{Implementation of HPSO and ETOPSIS method}

In this section, we propose a novel method by combining the HPSO algorithm and the ETOPSIS method to solve the multi-objective optimization problem for the EV charging station planning. The flow chart of the planning process is shown in Fig. 4.

\section{HPSO algorithm}

It is known that PSO is an evolutionary computation algorithm, where each particle represents a candidate solution of the considered problem and moves in a $D$-dimensional search space at a certain speed. The position and velocity of the $i$ th particle at the $t$ th iteration are denoted by two vectors, i.e., the position vector $X_{i}^{t}=\left[x_{i 1}^{t}, x_{i 2}^{t}, \ldots, x_{i D}^{t}\right]$ and the velocity vector $V_{i}^{t}=\left[v_{i 1}^{t}, v_{i 2}^{t}, \ldots, v_{i D}^{t}\right]$. In addition, $X_{i}^{t} \in\left[x_{\min }, x_{\max }\right]$, where $x_{\min }$ and $x_{\max }$ are, respectively, the lower and upper bounds for the particles' positions. $V_{i}^{t} \in\left[v_{\min }, v_{\max }\right]$, where $v_{\min }$ and $v_{\max }$ are, respectively, the lower and upper bounds for the particles' velocities. The velocity and position of the particle at the $(t+1)$ th iteration

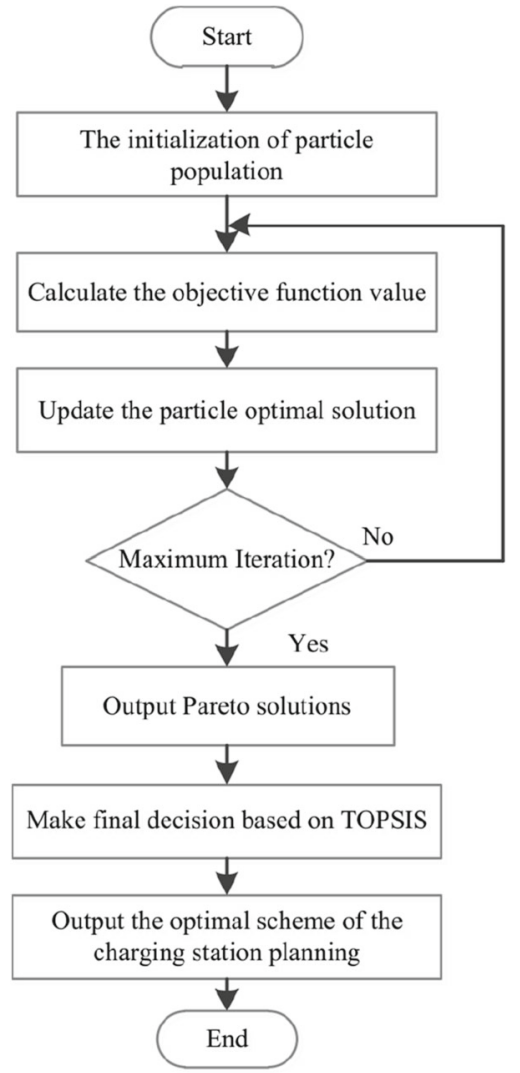

Fig. 4 Flowchart of the planning process

are updated according to the following equations:

$\left\{\begin{array}{l}v_{i j}^{t+1}=v_{i j}^{t}+c_{1} r_{1}\left(p_{i j}^{t}-x_{i j}^{t}\right)+c_{2} r_{2}\left(g_{i j}^{t}-x_{i j}^{t}\right) \\ x_{i j}^{t+1}=x_{i j}^{t}+v_{i j}^{t+1},\end{array}\right.$

where $t$ is the current iteration number, $c_{1}$ and $c_{2}$ are the acceleration coefficients called cognitive and social parameters, respectively. In addition, $r_{1}$ and $r_{2}$ are two random numbers, which are uniformly distributed in $[0,1] . p_{i j}^{t}$ is the local best position at the $t$ th iteration, and $g_{i j}^{t}$ is the global best position in the swarm at the $t$ th iteration.

Furthermore, as shown in (13), two acceleration coefficients $c_{1}$ and $c_{2}$ have a significant effect on the particles' motion. When $c_{1}$ is larger than $c_{2}$, the particles would mainly follow their own experience, which may result in the slower convergence speed. If $c_{2}$ is larger than $c_{1}$, the particles may be trapped in the local optimum location due to the faster convergence speed. To solve this problem, the PSO algorithm with constriction factor (PSOCF) has been proposed by Clerc and Kennedy in [6], which is able to balance the local and global optimum values by choosing the appropriate parameters, and the velocity formula is updated by

$v_{i j}^{t+1}=\varphi\left\{v_{i j}^{t}+c_{1} r_{1}\left(p_{i j}^{t}-x_{i j}^{t}\right)+c_{2} r_{2}\left(g_{i j}^{t}-x_{i j}^{t}\right)\right\}$, 
where $\varphi$ is a constriction factor defined as $\varphi=\frac{2}{\left|2-C-\sqrt{C^{2}-4 C}\right|}$ with $C=c_{1}+c_{2}$ and $C>4$. The constriction factor can effectively control the particles' moving speed and enhance the local searching ability of the algorithm. Nevertheless, with the increase of the number of iterations, particles might become similar and the search would fall into the local optimum. As such, the crossover and mutation operations of genetic algorithm (GA) are added into PSOCF to improve the particle diversity. The procedure of the HPSO is described as follows:

1. Initialize the HPSO algorithm by inputting EVs' travel information, population size (xSize), the particle length (Dim) and the maximum number of iterations (MaxIt).

2. Set the iteration counter $t=1$.

3. Generate the initial particle positions $X_{i}^{1}=\left[x_{i 1}^{1}, x_{i 2}^{1}\right.$, $\left.\ldots, x_{i n}^{1}\right]$ and velocities $V_{i}^{1}=\left[v_{i 1}^{1}, v_{i 2}^{1}, \ldots, v_{i n}^{1}\right]$ randomly. Each particle has $n$ components, and each component represents the node number of a charging station.

4. Predict the charging demand and calculate the values of the three objective functions for all particles.

5. Update the local best solution, the global best solution and the Pareto solution.

6. Update the velocities and positions of the particles with performing crossover and mutation operations on the particles.

7. Repeat steps 3-6 until the maximum number of iterations is reached.

\section{ETOPSIS method}

The technique for order preference by similarity to ideal solution (TOPSIS) is a systematic evaluation method that is suitable for the multi-criteria decision analysis [18,21], and the Entropy weight method is one of the most famous weighting methods, which can be employed to determine the objective weight of each dimension of available indexes [12]. In this paper, the ETOPSIS method is used to calculate the integrated closeness of each scheme, which facilitates to select the optimal scheme according to the rank of the closeness of candidate schemes.

\section{(1) Building standardized decision matrix}

In this stage, the charging station planning problem is converted into a decision problem with $l$-program and 3 -index. The standardized matrix is represented by

$T=\left[\tau_{i j}\right]_{l \times 3}, i=1,2, \ldots, l, j=1,2,3$ where $\tau_{i j}$ denotes the standardized value of the $j$ th index in the $i$ th scheme, and $l$ is the number of candidate schemes.

\section{(2) Calculating the entropy weight}

The entropy value of the $j$ th index is defined as

$e_{j}=-\frac{1}{\ln (l)} \sum_{i=1}^{l} p_{i j} \ln \left(p_{i j}\right)$

where $p_{i j}=\tau_{i j} / \sum_{i=1}^{l} \tau_{i j}$, and $p_{i j}$ denotes the proportion of index characteristics. $p_{i j} \ln \left(p_{i j}\right)=0$ when $p_{i j}=0$ or $p_{i j}=1$. Then, the entropy weight is calculated by

$w_{j}=\frac{1-e_{j}}{\sum_{j=1}^{3}\left(1-e_{j}\right)}$,

where $\sum_{j=1}^{3} w_{j}=1$.

\section{(3) Building weighted decision matrix}

Based on the standardized decision matrix and the entropy weight values, the weighted decision matrix is constructed as

$H=\left(h_{i j}\right)_{l \times 3}=\left(w_{j} \tau_{i j}\right)_{l \times 3}$.

\section{(4) Seeking the positive ideal solution and the negative ideal} solution

The positive ideal solution and the negative ideal solution are presented by

$\left\{\begin{array}{l}S_{j}^{+}=\left\{\left(\max h_{i j} \mid j \in J_{1}\right),\left(\min h_{i j} \mid j \in J_{2}\right) \mid i=1,2, \ldots l\right\} \\ S_{j}^{-}=\left\{\left(\min h_{i j} \mid j \in J_{1}\right),\left(\max h_{i j} \mid j \in J_{2}\right) \mid i=1,2, \ldots l\right\},\end{array}\right.$

where $J_{1}$ is the set of indexes that the bigger the index is, the better the scheme is, and $J_{2}$ is the set of indexes that the smaller the index is, the better the scheme is.

(5) Calculating the distance between candidate scheme and positve/negative ideal solution

For the $i$ th scheme, the distance with the positve/negative ideal solution is described by

$\left\{\begin{array}{l}S d_{i}^{+}=\sqrt{\sum_{j=1}^{3}\left(S_{j}^{+}-h_{i j}\right)^{2}} \\ S d_{i}^{-}=\sqrt{\sum_{j=1}^{3}\left(S_{j}^{-}-h_{i j}\right)^{2}} .\end{array}\right.$

\section{(6) Calculating the integrated closeness}

The integrated closeness of the $i$ th candidate scheme is defined as

$\sigma_{i}=\frac{S d_{i}^{-}}{S d_{i}^{+}+S d_{i}^{-}}$ 


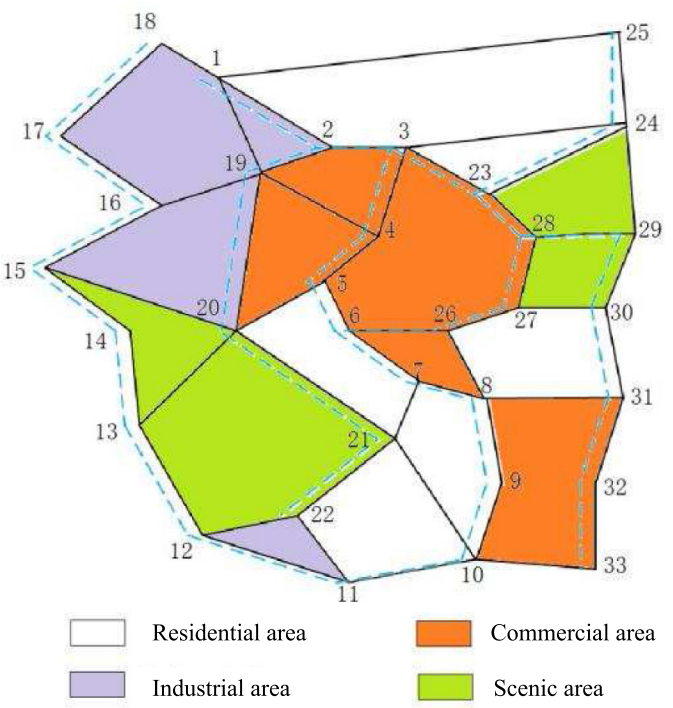

Fig. 5 Test system with an IEEE 33-node distribution system and a 33-node traffic network

Note that the bigger $\sigma_{i}$ is, the better the $i$ th scheme is. Thus, the closeness of candidate schemes can be ranked to obtain the optimal scheme.

\section{Case studies}

\section{Test system}

In this paper, a test system consisting of an IEEE 33-node distribution system and a 33-node traffic network is utilized to verify the effectiveness of the proposed method. It is assumed that the two kinds of nodes are geographically overlapped, as shown in Fig. 5, where the solid lines constitute the traffic network and the dotted lines constitute the power distribution system. The planning area is divided into residential area, industrial area, commercial area, and scenic area. The line parameters of the 33-node traffic network are shown in Table 1. Since the large-scale application of EVs is still in the early stage, the travelling information of existing fuel vehicles, which is taken from the Beijing Transport Annual Report in 2015[41], is utilized in this paper for analysis. Moreover, it is assumed that there are $5000 \mathrm{EVs}$ in the test system, and the technical parameters of EV refer to the parameters of BYD-E6. The battery capacity of EV is 82 $\mathrm{kW} \cdot \mathrm{h}$, and the average capacity consumption per kilometer is $21.5 \mathrm{~kW} \cdot \mathrm{h} / 100 \mathrm{~km}$. The EV driving range is up to 300 $\mathrm{km}$, and the EV average velocity is $40 \mathrm{~km} / \mathrm{h}$. The SOC of BYD-E6 is generally between 0.2 and 0.8 . In addition, the initial SOC of EV is assumed to obey the normal distribution $\mathcal{N}(0.6,0.1)$. The EV battery can be fully charged in 15 minutes by the fast charging mode, and in 4 hours by the
Table 1 Line parameters of the 33-node traffic network

\begin{tabular}{|c|c|c|c|c|c|}
\hline \multicolumn{3}{|c|}{ Line parameters } & \multicolumn{3}{|c|}{$\underline{\text { Line parameters }}$} \\
\hline $\mathrm{O}$ & $\mathrm{D}$ & Distance $/ \mathrm{km}$ & $\mathrm{O}$ & $\mathrm{D}$ & Distance $/ \mathrm{km}$ \\
\hline 1 & 2 & 16.6 & 12 & 13 & 27.9 \\
\hline 1 & 18 & 14.4 & 12 & 22 & 20.3 \\
\hline 1 & 19 & 18.6 & 13 & 14 & 21.7 \\
\hline 1 & 25 & 43.7 & 13 & 20 & 22.4 \\
\hline 2 & 3 & 13.9 & 14 & 15 & 23.1 \\
\hline 2 & 19 & 15.1 & 15 & 16 & 30.5 \\
\hline 3 & 4 & 19.4 & 15 & 20 & 33.6 \\
\hline 3 & 23 & 20.1 & 16 & 17 & 29.3 \\
\hline 3 & 24 & 33.8 & 16 & 19 & 19.3 \\
\hline 4 & 5 & 17.3 & 17 & 18 & 32.1 \\
\hline 4 & 19 & 18.7 & 19 & 20 & 20.8 \\
\hline 5 & 6 & 10.2 & 20 & 21 & 35.8 \\
\hline 5 & 20 & 19.9 & 21 & 22 & 24.7 \\
\hline 6 & 7 & 11.1 & 23 & 24 & 27.1 \\
\hline 6 & 26 & 12.7 & 23 & 28 & 13.9 \\
\hline 7 & 8 & 13.5 & 24 & 25 & 20.5 \\
\hline 7 & 21 & 15.8 & 24 & 29 & 22.2 \\
\hline 8 & 9 & 14.9 & 26 & 27 & 13.9 \\
\hline 8 & 26 & 12.8 & 27 & 28 & 14.1 \\
\hline 8 & 31 & 20.8 & 27 & 30 & 25.8 \\
\hline 9 & 10 & 15.5 & 28 & 29 & 19.2 \\
\hline 10 & 11 & 25.4 & 29 & 30 & 15.8 \\
\hline 10 & 21 & 28.5 & 30 & 31 & 18.9 \\
\hline 11 & 12 & 28.2 & 31 & 32 & 19.3 \\
\hline 11 & 22 & 18.3 & 32 & 33 & 20.3 \\
\hline
\end{tabular}

conventional charging mode. The fast charging power is set to be $96 \mathrm{~kW}$ and the conventional charging power is set to be $5 \mathrm{~kW}$. The charging rate is set to be $80 \%$.

\section{Simulation results of the proposed method}

In the simulation, the population size of the HPSO algorithm is $x$ Size $=50$, the particle length is Dim $=10$ and the maximum number of iterations is MaxIt $=50$. In addition, the number of charging stations is 10 , which is equal to the particle length. The optimization results (e.g. the optimized values of three objective functions) from the HPSO algorithm are used in the ETOPSIS method to get evaluation indicators, and the entropy weights of three indicators are derived as $0.33688,0.291538$ and 0.371586 . The specific values and closeness coefficients of Pareto solutions are shown in Table 2. It can be seen that the closeness of the first scheme is about 0.58655 , which is the largest one and meets the decision requirement. Then, the layout of charging stations is determined by choosing the optimal scheme, 
Table 2 Pareto solution set and TOPSIS decision results

\begin{tabular}{lllll}
\hline Index & Captured demand $(\mathrm{kW})$ & Total costs $(¥)$ & Load variance $\left(\mathrm{kW}^{2}\right)$ & Closeness \\
\hline 1 & $1,091,594.65$ & $597,100.88$ & $548,324,346.10$ & 0.58655 \\
2 & $870,085.50$ & $827,686.46$ & $668,123,391.72$ & 0.56905 \\
3 & $782,335.88$ & $620,690.65$ & $677,319,491.18$ & 0.541848 \\
4 & $707,374.01$ & $663,874.33$ & $672,430,154.62$ & 0.437017 \\
5 & $658,234.24$ & $532,507.29$ & $570,283,068.54$ & 0.377016 \\
6 & $691,148.23$ & $512,309.68$ & $667,137,074.93$ & 0.355856 \\
7 & $867,709.42$ & $687,180.50$ & $545,358,826.05$ & 0.347902 \\
8 & $848,258.17$ & $682,316.83$ & $646,291,941.96$ & 0.328652 \\
\hline
\end{tabular}

which is shown in Table 3. It is worth mentioning that even though the cost and load variance of some schemes are less than that of the first scheme, they are not adopted due to the lower closeness.

In addition, Figs. 6 and 7 show, respectively, the temporal and spatial distributions of EVs' charging demand. It can be seen from Fig. 6 that EV users prefer to choose the fast charging mode during the morning/afternoon peak hours, and choose the conventional charging mode during the off-peak hours. Figure 7 depicts the demands of fast charging and conventional charging in different charging stations. It is clear that the demands of fast charging and conventional charging vary with the location of charging station. For instance, since charging station 8 is located in the junction of two residential areas and two commercial areas, the demands of both charging modes are relatively high. Moreover, charging stations $3,11,21$, and 25 are close to residential areas, therefore the demand of conventional charging mode is higher than that of the fast charging mode. Charging station 15, 18, and 28 are close to the industrial and commercial areas, the demand of conventional charging mode are lower than that of fast charging mode. Furthermore, the specific configuration of fast charger and conventional charger in the charging stations is shown in Table 3, which corresponds to the charging demands under the optimal scheme described in Fig. 7.

\section{Effectiveness of charging station planning with different EV charging behaviors}

In section "Dynamic EV charging demand forecasting", the satisfaction function has been presented to help EV users to select the appropriate charging stations, which also facilitates the efficient planning of charging stations in the functional region. To further reveal the feasibility of the proposed method, the effectiveness analysis of charging station planning is conducted with the following three types of $\mathrm{EV}$ charging behaviors:

Case 1: The charging station is selected according to the satisfaction degree of EV users presented in this paper.

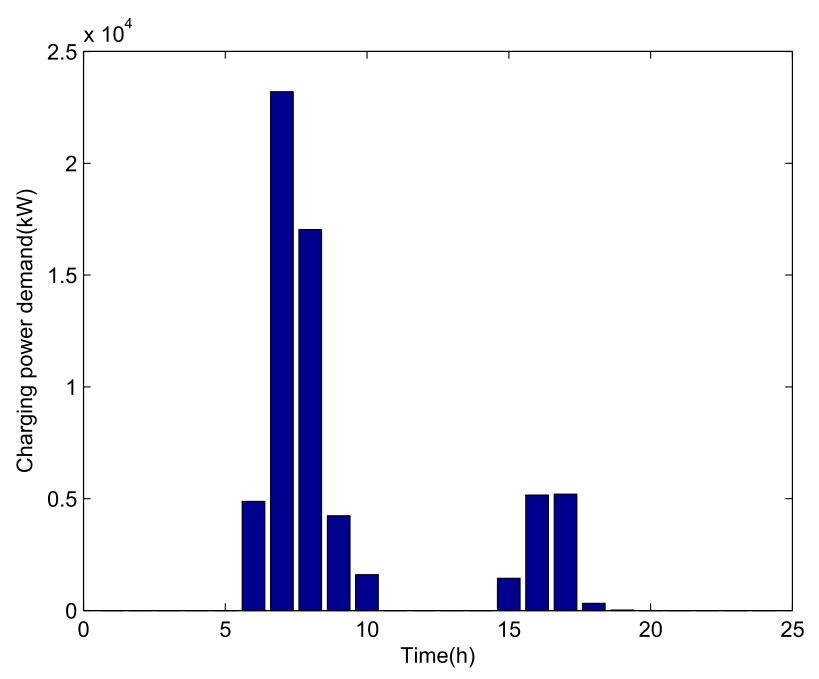

(a)

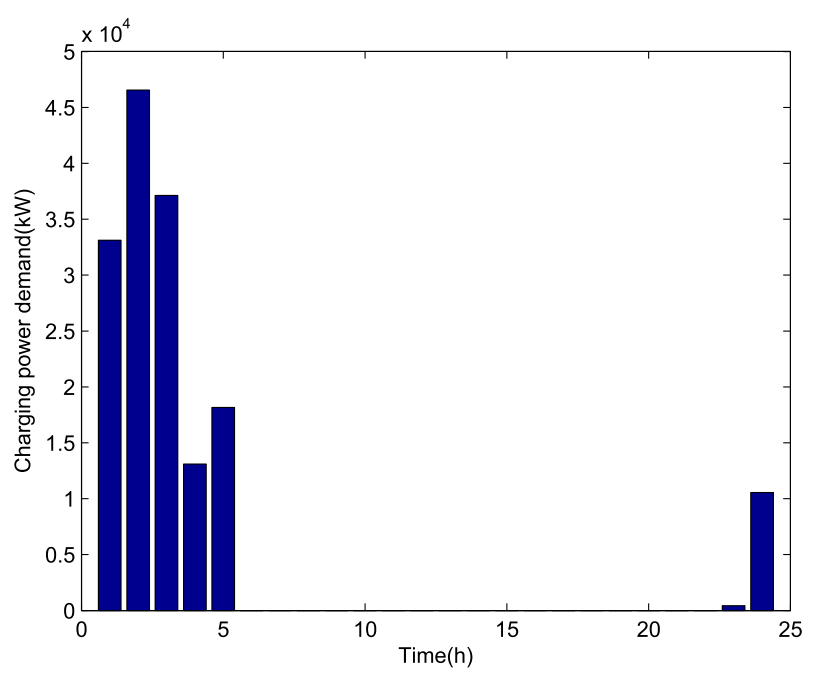

(b)

Fig. 6 The temporal distribution of charging demand under the optimal layout scheme. a Fast charging. b Conventional charging

Case 2: The charging station is selected according to the shortest distance between the charging station and EV users. 


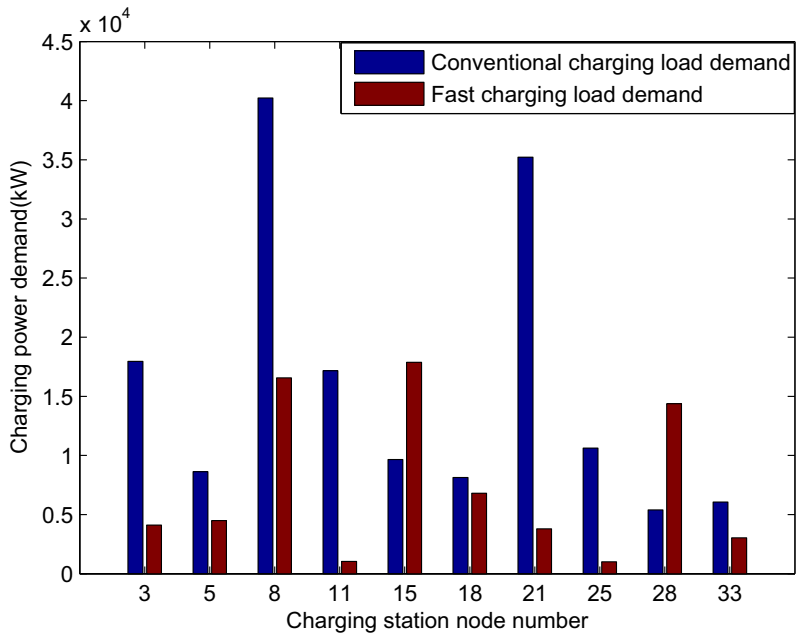

Fig. 7 The spatial distribution of charging demand under the optimal layout scheme

Case 3: The charging station is selected according to individual random preference of $\mathrm{EV}$ users in the driving process.

The results for Cases 1, 2, and 3 are given in Table 4. It can be seen from Table 4 that the scheme proposed in this paper, corresponding to Case 1, has the maximum captured demands, the minimum extra cost, and the minimum load variance as expected. In Case 3, the captured demands are much higher than that in Case 2, but the extra charging cost and load variance are higher than that in Case 1 and Case 2. Therefore, the establishment of an appropriate satisfaction function that takes into account the actual charging requirements can improve the planning and operation efficiency of charging stations.

Remark 2 In this paper, the EV travel characteristics, charging costs and stable operation of power grid have been fully taken into account in planning the charging stations. More specifically, a multi-objective charging station planning problem has been formulated to achieve three objectives, namely, maximizing the captured charging demands, minimizing the extra charging cost, and minimizing the load variance of the power grid. The optimal scheme is actually a compromise between these three objectives. As shown in Table 2, the optimal scheme has the maximum captured charging demand, however, the costs and the load variance are not the minimum among all candidate schemes, which implies that a rational tradeoff has been obtained among different concerns. Furthermore, it should be mentioned that the proposed scheme is able to alleviate the traffic pressure caused by the EV charging behavior, enhance the charging convenience of EV users, and guarantee the stability of grid operation.

\section{Conclusion}

In this paper, a novel method has been proposed to solve the EV charging station planning problem based on the dynamic prediction of charging demand. Considering the relationship between the EV charging demand and the individual driving habits, the dynamic forecasting method of charging demand has been developed by virtue of the analysis on EV users' travel laws. In addition, a multi-objective planning model has been proposed by considering the EV travel characteristics, charging costs and stable operation of power grid. The capacity configuration model has also been established according to the different demands of fast charging mode and conventional charging mode. Moreover, the HPSO algorithm has been utilized to obtain the Pareto solutions and the ETOPSIS method has been employed to determine the optimal planning scheme. Based on the proposed method, the optimal siting and sizing of EV charging station have been achieved to maximize the captured charging demands, minimize the extra cost, and minimize the load variance of the power grid. Finally, the case studies have demonstrated that the proposed method can attain the reasonable planning of the EV charging stations. Further research topics include (1) the EVs charging demand analysis based on the K-means technique [1] and the reinforcement learning method [8]; (2) the estimation of EV SOC based on the measurement

Table 3 Capacity configuration under the optimal scheme

\begin{tabular}{lrrrrrrrrrr}
\hline Charging station node number & 3 & 5 & 8 & 11 & 15 & 18 & 21 & 25 & 28 & 33 \\
The number of fast charger & 13 & 14 & 35 & 5 & 38 & 16 & 11 & 5 & 32 & 10 \\
The number of conventional charger & 106 & 60 & 220 & 98 & 65 & 52 & 176 & 70 & 42 & 45 \\
\hline
\end{tabular}

Table 4 Effectiveness analysis of different EV charging behaviors

\begin{tabular}{lllll}
\hline Case number & Sitting scheme & Captured demand $(\mathrm{kW})$ & Total costs $(¥)$ & Load variance $(\mathrm{kW})$ \\
\hline 1 & 35811151821252833 & 1091594.65 & 597100.88 & 548324346.10 \\
2 & 14721222526273032 & 852579.77 & 823447.26 & 638399360.16 \\
3 & 730121131814281924 & 1030191.70 & 984149.64 & 672736341.75 \\
\hline
\end{tabular}


with the network induced phenomenon [4,31,32,37]; (3) the EV charging station planning problem subject to incomplete information $[19,23,25,28,38,43]$; (4) the cooperative control of multiple vehicles based on the multiagent systems [7]; and (5) the optimization problem with the weighted modularity optimization approach [2].

Acknowledgements This work was supported in part by the National Natural Science Foundation of China under Grants 61703245, 61873148 and 61933007, the Natural Science Foundation of Shandong Province of China under Grant ZR2020MF071, the AHPU Youth Top-notch Talent Support Program of China under Grant 2018BJRC009, the Natural Science Foundation of Anhui Province of China under Grant 2108085MA07, the Royal Society of the UK, and the Alexander von Humboldt Foundation of Germany.

\section{Declarations}

Conflict of interest On behalf of all authors, the corresponding author states that there is no conflict of interest.

Open Access This article is licensed under a Creative Commons Attribution 4.0 International License, which permits use, sharing, adaptation, distribution and reproduction in any medium or format, as long as you give appropriate credit to the original author(s) and the source, provide a link to the Creative Commons licence, and indicate if changes were made. The images or other third party material in this article are included in the article's Creative Commons licence, unless indicated otherwise in a credit line to the material. If material is not included in the article's Creative Commons licence and your intended use is not permitted by statutory regulation or exceeds the permitted use, you will need to obtain permission directly from the copyright holder. To view a copy of this licence, visit http://creativecomm ons.org/licenses/by/4.0/.

\section{References}

1. Bu Z, Li H-J, Zhang C, Cao J, Li A, Shi Y (2019) Graph K-means based on leader identification, dynamic game and opinion dynamics. IEEE Trans Knowl Data Eng 32(7):1348-1361

2. Cao J, Bu Z, Gao G, Tao H (2016) Weighted modularity optimization for crisp and fuzzy community detection in large-scale networks. Physica A 462:386-395

3. Chen J, Ai Q, Xiao F (2016) EV charging station planning based on travel demand. Electr Power Autom Equip 36(6):34-39

4. Chen Y, Chen Z, Chen Z, Xue A (2020) Observer-based passive control of non-homogeneous Markov jump systems with random communication delays. Int J Syst Sci 51(6):1133-1147

5. Chen T, Wei Z, Wu S, Sun G, Wei Y, Xu X (2013) Distribution network planning by considering siting and sizing of electric vehicle charging stations. Proc CSU-EPSA 25(3):1-7

6. Clerc M, Kennedy J (2002) The particle swarm-explosion, stability, and convergence in a multidimensional complex space. IEEE Trans Evol Comput 6(1):58-73

7. Cui Y, Liu Y, Zhang W, Alsaadi FE (2021) Sampled-based consensus for nonlinear multiagent systems with deception attacks: the decoupled method. IEEE Trans Syst Man Cybern Syst 51(1):561573

8. Ding D, Ding Z, Wei G, Han F (2019) An improved reinforcement learning algorithm based on knowledge transfer and applications in autonomous vehicles. Neurocomputing 361:243-255
9. Goudarzi H, Hatami S, Pedram M (2011) Demand-side load scheduling incentivized by dynamic energy prices. In: Proceedings of the 2011 IEEE International Conference on Smart Grid Communications, pp 351-356, Brussels

10. Hu J, Zhang H, Liu H, Yu X (2021) A survey on sliding mode control for networked control systems. Int J Syst Sci 52(6):1129_ 1147

11. Hu S, Yue D, Han Q-L, Xie X, Chen X, Dou C (2020) Observerbased event-triggered control for networked linear systems subject to denial-of-service attacks. IEEE Trans Cybern 50(5):1952-1964

12. Huang W, Shuai B, Yan S, Wang Y, Antwi E (2018) Using entropyTOPSIS method to evaluate urban rail transit system operation performance: the China case. Transp Res Part A Policy Pract 111:292-303

13. Ikegami T, Ogimoto K, Yano H, Kudo K, Iguchi H (2012) Balancing power supply-demand by controlled charging of numerous electric vehicles. In: Proceedings of the 2012 IEEE international electric vehicle conference, Greenville

14. Ismail M, Bayram IS, Abdallah M, Sperpedin E, Qaraqe K (2015) Optimal planning of fast PEV charging facilities, 2015 First Workshop on Smart Grid and Renewable Energy (SGRE). Doha, Qatar

15. Jia L, Hu Z, Liang W, Tang W, Song Y (2014) A novel approach for urban electric vehicle charging facility planning considering combination of slow and fast charging. In: Proceedings of the 2014 International Conference on Power System Technology, Chengdu, pp 3354-3360

16. Kang Q, Feng S, Zhou M, Ammari AC, Sedraoui K (2017) Optimal load scheduling of plug-in hybrid electric vehicles via weightaggregation multi-objective evolutionary algorithms. IEEE Trans Intell Transp Syst 18(9):2557-2668

17. Korkas CD, Baldi S, Yuan S, Kosmatopoulos EB (2018) An adaptive learning-based approach for nearly optimal dynamic charging of electric vehicle fleets. IEEE Trans Intell Transp Syst 19(7):20662075

18. Li D-F (2010) TOPSIS-based nonlinear-programming methodology for multiattribute decision making with interval-valued intuitionistic fuzzy sets. IEEE Trans Fuzzy Syst 18(2):299-311

19. Li Q, Liang J (2020) Dissipativity of the stochastic Markovian switching CVNNs with randomly occurring uncertainties and general uncertain transition rates. Int J Syst Sci 51(6):1102-1118

20. Li X, Han F, Hou N, Dong H, Liu H (2020) Set-membership filtering for piecewise linear systems with censored measurements under Round-Robin protocol. Int J Syst Sci 51(9):1578-1588

21. Liu H-C, Wang L-E, Li Z, Hu Y-P (2019) Improving risk evaluation in FMEA with cloud model and hierarchical TOPSIS method. IEEE Trans Fuzzy Syst 27(1):84-95

22. Liu J, Gu Y, Zha L, Liu Y, Cao J (2019) Event-triggered $H_{\infty}$ load frequency control for multiarea power systems under hybrid cyber attacks. IEEE Trans Syst Man Cybern Syst 49(8):1665-1678

23. Liu L, Ma L, Zhang J, Bo Y (2021) Distributed non-fragile setmembership filtering for nonlinear systems under fading channels and bias injection attacks. Int J Syst Sci 52(6):1192-1205

24. Liu Y, Shen B, Shu H (2020) Finite-time resilient $H_{\infty}$ state estimation for discrete-time delayed neural networks under dynamic event-triggered mechanism. Neural Netw 121:356-365

25. Liu Y, Shen B, Li Q (2019) State estimation for neural networks with Markov-based nonuniform sampling: The partly unknown transition probability case. Neurocomputing 357:261-270

26. Liu Y, Cheng Q, Gan Y, Wang Y, Li Z, Zhao J (2019) Multiobjective optimization of energy consumption in crude oil pipeline transportation system operation based on exergy loss analysis. Neurocomputing 332:100-110

27. Liu Y, Chen S, Guan B, Xu P (2019) Layout optimization of largescale oil-gas gathering system based on combined optimization strategy. Neurocomputing 332:159-183 
28. Mao J, Sun Y, Yi X, Liu H, Ding D (2021) Recursive filtering of networked nonlinear systems: a survey. Int J Syst Sci 52(6):1110 1128

29. Peng C, Yue D, Fei MR (2016) A higher energy-efficient sampling scheme for networked control systems over IEEE 802.15.4 wireless networks. IEEE Trans Ind Inf 12(5):1766-1774

30. Phonrattanasak P, Leeprechanon N (2014) Multi-objective ant colony optimization for fast charging stations planning in residential area. In: Proceedings of the 2014 IEEE Innovative Smart Grid Technologies-Asia (ISGT ASIA), Kuala Lumpur, pp 290-295

31. Qian W, Li Y, Zhao Y, Chen Y (2020) New optimal method for $L_{2}$ $L_{\infty}$ state estimation of delayed neural networks. Neurocomputing 415:258-265

32. Qian W, Li Y, Chen Y, Liu W (2020) $L_{2}-L_{\infty}$ filtering for stochastic delayed systems with randomly occurring nonlinearities and sensor saturation. Int J Syst Sci 51(13):2360-2377

33. Ren Y, Shi L, Zhang Q, Han W, Huang S (2011) Optimal distribution and scale of charging station for electric vehicles. Autom Electric Power Syst 35(14):53-57

34. Silva DF, Abud TP, Jr PPM, Fortes MZ, Maciel RS, Dias DHN (2016) Impact analysis of plug-in electric vehicle connected in real distribution network. IEEE Lat Am Trans 14(5):2239-2244

35. Sortomme E, Hindi MM, MacPherson SDJ, Venkata SS (2011) Coordinated charging of plug-in hybrid electric vehicles to minimize distribution system losses. IEEE Trans Smart Grid 2(1):198205

36. Sun J, Wan Y, Zheng P, Lin X (2014) Coordinated charging and discharging strategy for electric vehicles based on demand side management. Trans Chin Electrotech Soc 29(8):64-69

37. Sun Y, Mao J, Liu H, Ding D (2020) Distributed recursive filtering for discrete time-delayed stochastic nonlinear systems based on fuzzy rules. Neurocomputing 400:412-419

38. Tan H, Shen B, Peng K, Liu H (2020) Robust recursive filtering for uncertain stochastic systems with amplify-and-forward relays. Int J Syst Sci 51(7):1188-1199

39. Tao S, Liao K, Xiao X, Wen J, Yang Y, Zhang J (2016) Charging demand for electric vehicle based on stochastic analysis of trip chain. IET Gen Trans Distrib 10(11):2689-2698
40. Wang G, Zhang X, Wang H, Peng J-C, Jiang H, Liu Y, Wu C, Xu Z, Liu W (2018) Robust planning of electric vehicle charging facilities with an advanced evaluation method. IEEE Trans Ind Inf 14(3):866-876

41. Wen H, Liu Y, Du H et al (2015) Beijing Transport Annual Report in 2015. Beijing Traffic Development Research Center, Beijing

42. Xue Y, Wu J, Xie D, Li K, Zhang Y, Wen F, Cai B, Wu Q, Yang G (2015) Multi-agents modelling of EV purchase willingness based on questionnaires. J Mod Power Syst Clean Energy 3(2):149-159

43. Yang F, Han Q-L, Liu Y (2019) Distributed $H_{\infty}$ state estimation over a filtering network with time-varying and switching topology and partial information exchange. IEEE Trans Cybern 49(3):870882

44. Yue X, Liu J, Li R, Li F, Gu C, Tang S (2016) Economic planning of electric vehicle charging stations considering traffic constraints and load profile templates. Appl Energy 178:647-659

45. Zhang J, Feng Y, Shi F, Wang G, Ma B, Li R, Jia X (2016) Vehicle routing in urban areas based on the Oil consumption weightDijkstra algorithm. IET Intel Transp Syst 10(7):495-502

46. Zhang H, Moura SJ, Hu Z, Song Y (2018) PEV fast-charging station siting and sizing on coupled transportation and power networks. IEEE Trans Smart Grid 9(4):2595-2605

47. Zhou Y, Xu G (2014) Demand side energy management with PSO and regulated electric vehicles behaviours. In: 2014 IEEE PES Asia-Pacific Power and Energy Engineering Conference (APPEEC). China, Hong Kong

48. Zhu K, Hu J, Liu Y, Alotaibi ND, Alsaadi FE (2021) On $\ell_{2}-\ell_{\infty}$ output-feedback control scheduled by stochastic communication protocol for two-dimensional switched systems. Int J Syst Sci 52(14):2961-2976

Publisher's Note Springer Nature remains neutral with regard to jurisdictional claims in published maps and institutional affiliations. 